\title{
EchoGéo
}

$6 \mid 2008$

La Guyane

\section{Gros temps}

Jean Marie Théodat

\section{OpenEdition}

Journals

Édition électronique

URL : https://journals.openedition.org/echogeo/7743

DOI : $10.4000 /$ echogeo.7743

ISSN : 1963-1197

\section{Éditeur}

Pôle de recherche pour l'organisation et la diffusion de l'information géographique (CNRS UMR 8586)

Référence électronique

Jean Marie Théodat, «Gros temps », EchoGéo [En ligne], 6 | 2008, mis en ligne le 07 octobre 2008, consulté le 31 juillet 2021. URL : http://journals.openedition.org/echogeo/7743 ; DOI : https://doi.org/ $10.4000 /$ echogeo.7743

Ce document a été généré automatiquement le 31 juillet 2021.

EchoGéo est mis à disposition selon les termes de la licence Creative Commons Attribution - Pas d'Utilisation Commerciale - Pas de Modification 4.0 International (CC BY-NC-ND) 


\title{
Gros temps
}

\author{
Jean Marie Théodat
}

1 Ce sixième cahier paraît à un moment où « l'immense orgue des vents grondeurs » cher à Baudelaire fait rage dans les finances du pays le plus puissant de la planète. Les turbulences provoquées par l'explosion de la bulle financière américaine entraînent un déséquilibre mondial qui va demander des réajustements structurels et profonds dans le modèle de développement du pays le plus riche. Les fondements même de l'économie libérale se trouvent ébranlés dans le pays qui en a fait son credo et son mode de conquête des esprits et des marchés dans le monde. Le principe de libre circulation des idées, des capitaux et des marchandises, à la base de l'idéal occidental est aujourd'hui en panne. En ces temps de mondialisation tous azimuts, le retour à l'Etat, implicite dans l'adoption aux Etats-Unis du plan Paulson en vue du sauvetage des établissements financiers privés menacés de faillite, vaut désaveu de la capacité du marché à fonctionner par autorégulation sans interventions, sous une forme ou sous une autre, des pouvoirs publics.

2 L'actualité tempétueuse du monde de la finance ne doit pas nous faire oublier d'autres gros temps. Ceux qui ont frappé la Caraïbe et les pays de l'océan Indien durant la saison cyclonique 2008 furent parmi les plus meurtriers de ces dernières années et depuis que les instruments de mesure permettent d'évaluer à la fois l'ampleur des dégâts, le nombre des victimes et l'intensité du phénomène. Le classement sur l'échelle de SaffirSimpson fait apparaitre une énergie grandissante des cyclones les plus importants (plus de $250 \mathrm{~km} / \mathrm{h}$ ) depuis une vingtaine d'années, correspondant à une élévation de $0,3^{\circ} \mathrm{C}$ de la température moyenne des eaux de surface des régions cycloniques entre 1981 et 2006. Les noms de Gustav, Hannah, Ike, ont rejoint ceux de "monstres» comme Katrina, Wilma, pour les plus récents, Flora et Hazel, parmi les plus anciens, etc. dans les annales de la météorologie tropicale. Notre rubrique Sur le Terrain revient sur cette actualité cyclonique avec un article de Sandra Rome qui met en perspective une question de terrain, la recrudescence de l'activité cyclonique dans l'Atlantique Nord.

3 La rubrique Sur le Métier est l'occasion de suivre le parcours semé d'embûches et de chausse-trapes des doctorants dans la discipline géographique. Des doctorants. Le pluriel ici est de rigueur, le profil change d'un individu à l'autre. Entre Anthony 
Goreau-Ponceaud, Dan Liao, Laurence Buzenot, Fabrice Fussy, Elodie Briche, il n'y a de commun que la discipline où ils exercent leurs talents: la géographie. Car leurs témoignages diffèrent, voire parfois divergent. Non seulement par les thématiques abordées, les terrains choisis, les méthodologies adoptées, mais également par les conditions matérielles, le cadre et l'encadrement intellectuel du travail accompli. La relation avec le directeur de recherche, les perspectives d'emploi après la soutenance sont autant de thèmes évoqués à travers une série d'entretiens et d'échanges avec une diversité de géographes en herbe qui sont la promesse de moissons profuses d'idées nouvelles pour les années à venir.

4 Loin des gros vents coutumiers des latitudes tropicales et des pays insulaires, et des sautes d'humeur des thésards, la rubrique Sur le Champ, nous invite à explorer des horizons plus stables sinon plus clairs. Au plus proche de l'Equateur et d'un massif ancien aux sommets arrondis, comme endormis. En effet, la Guyane le plus grand Département Français d'Amérique, ne connaît ni de passage de cyclones, ni de turbulences financières impliquant l'avenir du reste de la planète. Mais ce pays paisible est appelé à devenir le point de convergence de la politique ultramarine la plus active de la France dans le Nouveau Monde.

5 Une certaine représentation de la Guyane l'associe volontiers à la condition des îles, ses voisines antillaises en général, la Martinique et la Guadeloupe en particulier. Ce n'est pas sans raison: une culture créole commune, une égale origine africaine de la majorité de la population et enfin l'appartenance au territoire français. Et pourtant il y a tant de différences qu'il faut se donner la peine d'une analyse plus approfondie pour faire apparaître la spécificité de cette terre encore largement inconnue. Car, au-delà des autres Départements Français d'Amérique, la Guyane confine à des territoires, des blocs régionaux qui l'inscrivent au cœur des grands bouleversements migratoires, environnementaux et économiques appelés à transformer les relations des hommes avec leurs territoires, en tant que réserves de richesses et limite de l'exercice de la souveraineté nationale. D'autres gros temps en perspective?

Pour en parler avec intelligence et débrouiller les perspectives de l'ouverture sur le reste de l'Amérique du Sud qu'implique pour la France, à travers ses frontières avec le Surinam et le Brésil, la valorisation croissante des richesses de cet territoire, nous avons réuni des spécialistes du terrain, géographes ou venus d'autres disciplines. Gérald Migeon, évoque les racines précolombiennes des civilisations guyanaises, en les associant aux trouvailles des fouilles archéologiques récentes. Stéphane Granger éclaire notre analyse sur la porosité croissante de la frontière avec le Brésil. André Calmont, dépiste les nouvelles sources et ressources de l'immigration en Guyane, brossant le panorama de ramifications de plus en plus fines selon des circuits de plus en plus complexes dans les trajectoires spatiales et sociales des migrants. A plus grande échelle et dans un pas de temps plus proche de l'évolution contemporaine, Isabelle Hidair et Pierre-Yves Chicot analysent l'évolution de l'île de Cayenne qui est, en quelque sorte, la région-coeur de ce département ultramarin.

7 La rubrique Sur l'Ecrit recense les nouveautés bibliographiques de la Bibliothèque de géographie et revient sur l'actualité d'une île quelque peu oubliée. Il s'agit de Zanzibar dont la singularité a autant trait à sa position insulaire qu'à sa situation de point d'articulation entre les aires africaines, persanes, indiennes et arabes dans ce carrefour de l'océan Indien. Autrefois plaque tournante du commerce des épices et des esclaves entre les mondes africains et moyen-orientaux, l'Autre Zanzibar est ici abordé du 
dedans, sous la plume de Nathalie Bernardie-Tahir, à travers la grille de ses relations complexes avec le reste de la fédération tanzanienne dont l'île fait partie tout en cherchant à préserver son originalité culturelle et administrative. La veille thématique à laquelle s'appliquent Alain Lebfèvre et Claire List nous dresse la carte du tendre des publications les plus intéressantes ayant trait aux aspects évoqués dans la rubrique Sur le Champ. 